\title{
ON ALGEBRAS OF RELATIONS
}

\author{
D. A. BREDIKHIN
}

Lermontova 7-22, 410002 Saratov, Russia

Throughout, by relation we mean a binary relation. Let $\operatorname{Rel}(X)$ be the set of all binary relations on the set $X$. An algebra of relations is a pair $(\Phi, \Omega)$ where $\Omega$ is a set of operations on relations and $\Phi \subset \operatorname{Rel}(X)$ is a set of relations closed under the operations of $\Omega$. Each algebra of relations can be considered as ordered by the set-theoretic inclusion $\subset$. Denote by $M\{\Omega\}$ the class of all algebras isomorphic to ones whose elements are relations and whose operations are members of $\Omega$. The class $M\{\Omega, \subset\}$ is determined in the same way.

We will consider the following operations on relations: relation product $\circ$, relation inverse ${ }^{-1}$, intersection $\cap$, diagonal relation $\Delta$, and the unary operation * determined as follows: $\varrho^{*}=\varrho \cap \Delta$.

The class $M\left\{\circ,^{-1}, \cap, \Delta\right\}$ was introduced and characterized in [6]. It is not finitely axiomatizable [5]. The classes $M\left\{0^{-1}, \Delta\right\}$ and $M\left\{0^{-1}, \Delta, \subset\right\}$ were characterized in [1,8]. The class $M\left\{0^{-1}, \Delta\right\}$ is not finitely axiomatizable [2].

In this paper we find a system of axioms for the class $M\left\{0^{-1},{ }^{*}, \Delta, \subset\right\}$ and use it to obtain some results about the class $M\left\{0^{-1}, \cap, \Delta\right\}$.

Theorem 1. An algebra $\left(A, \cdot,^{-1},{ }^{*}, 1, \leq\right)$ belongs to $M\left\{0,{ }^{-1},{ }^{*}, \Delta, \subset\right\}$ iff it satisfies the following conditions:

(1) $\left(A, \cdot,^{-1}, 1\right)$ is an involuted monoid, i.e. $(x y) z=x(y z), 1 x=x 1=x$ $\left(x^{-1}\right)^{-1}=x,(x y)^{-1}=y^{-1} x^{-1}$.

(2) $\leq$ is an order relation and all operations are monotonic, i.e. $x \leq y$ implies $x z \leq y z, z x \leq z y, x^{-1} \leq y^{-1}, x^{*} \leq y^{*}$.

(3) The following identities are satisfied:

$$
\left(x^{*}\right)^{*}=x^{*},
$$

1991 Mathematics Subject Classification: 03G15.

Key words and phrases: agebras of relations, representations, involuted semigroups.

The paper is in final form and no version of it will be published elsewhere. 


$$
\begin{gathered}
x^{*} x^{*}=x^{*}, \\
x^{*} y^{*}=y^{*} x^{*}, \\
\left(x^{*} y^{*}\right)^{*}=x^{*} y^{*}, \\
\left(x x^{-1}\right)^{*} x=x, \\
\left(x y y^{-1} x^{-1}\right)^{*}=\left(x\left(y y^{-1}\right)^{*} x^{-1}\right)^{*}, \\
x^{*} \leq 1, \\
x^{*} \leq x .
\end{gathered}
$$

Suppose that an algebra $\underline{A}=\left(A, \cdot,^{-1}, \wedge, 1\right) \in M\left\{0,{ }^{-1}, \cap, \Delta\right\}$. Then $\underline{A}$ is a semilattice ordered involuted monoid, i.e. $\left(A, \cdot,^{-1}, 1\right)$ is an involuted monoid, $\left(A, \wedge,{ }^{-1}\right)$ is an involuted semilattice and the identity $x(y \wedge z) \leq x y \wedge x z$ holds ( $\leq$ is the natural order of the semilattice) [6]. It is known [3] that $\underline{A}$ also satisfies

$$
x y \wedge z \leq\left(x \wedge y z^{-1}\right) y .
$$

We say that a semilattice ordered involuted monoid $\underline{A}$ is weakly representable if there exists a mapping $F: A \rightarrow \operatorname{Rel}(X)$ for some $X$ such that $F(a b)=$ $F(a) \circ F(b), F\left(a^{-1}\right)=F(a)^{-1}, F(1)=\Delta, F(a \wedge 1)=F(a) \cap \Delta$ and $a \leq b$ iff $F(a) \subset F(b)$ for all $a, b \in A$.

THEOREM 2. Suppose that a semilattice ordered involuted monoid satisfies (4). Then it is weakly representable.

The following condition plays an important role in applications of algebras of relations to logic [4]:

$$
(\exists u, v)(\forall a) u^{-1} u \leq 1 \& v^{-1} v \leq 1 \& a \leq u^{-1} v .
$$

Consider the condition

$$
(\forall a)(\exists u, v) u^{-1} u \leq 1 \& v^{-1} v \leq 1 \& a \leq u^{-1} v .
$$

Obviously, (5) implies (6).

THEOREM 3. Suppose that a semilattice ordered involuted monoid $\underline{A}$ satisfies (6). Then $\underline{A}$ belongs to $M\left\{0,,^{-1}, \cap, \Delta\right\}$ iff it satisfies (4).

Proof of Theorem 1. Necessity. Consider the ordered algebra of relations of the form $\left(\Phi, \circ,{ }^{-1},{ }^{*}, \Delta, \subset\right)$. It is well known that $\left(\Phi, \circ,^{-1}, \Delta\right)$ is an involuted monoid and the operations $\circ$ and ${ }^{-1}$ are monotonic $[1,8]$. Suppose that $\varrho, \pi \in \Phi$. Since $\varrho^{*}, \pi^{*} \subset \Delta$, we have $\varrho^{*} \circ \pi^{*}=\varrho^{*} \cap \pi^{*}$. It follows that (3.1)-(3.4) hold. If $\varrho \subset \pi$ then $\varrho^{*}=\varrho \cap \Delta \subset \pi \cap \Delta=\pi^{*}$, i.e. the operation * is monotonic. Since $\left(\varrho \circ \varrho^{-1}\right)^{*} \subset \Delta$, we have $\left(\varrho \circ \varrho^{-1}\right)^{*} \circ \varrho \subset \varrho$. Conversely, if $(x, y) \in \varrho$ then $(x, x) \in \varrho \circ \varrho^{-1} \cap \Delta=\left(\varrho \circ \varrho^{-1}\right)^{*}$ and $(x, y) \in\left(\varrho \circ \varrho^{-1}\right)^{*} \circ \varrho$, i.e. $\varrho \subset\left(\varrho \circ \varrho^{-1}\right)^{*} \circ \varrho$. Since $\left(\pi \circ \pi^{-1}\right)^{*} \subset \pi \circ \pi^{-1}$, we have $\left(\varrho \circ\left(\pi \circ \pi^{-1}\right)^{*} \circ \varrho^{-1}\right)^{*} \subset\left(\varrho \circ \pi \circ \pi^{-1} \circ \varrho^{-1}\right)^{*}$. Conversely, if $(x, x) \in\left(\varrho \circ \pi \circ \pi^{-1} \circ \varrho^{-1}\right)^{*}$ then there exist $y, z$ such that $(x, y) \in \varrho$ and $(y, z) \in \pi$, hence $(y, y) \in \pi \circ \pi^{-1} \cap \Delta=\left(\pi \circ \pi^{-1}\right)^{*}$ and $(x, y) \in\left(\varrho \circ\left(\pi \circ \pi^{-1}\right)^{*} \circ \varrho^{-1}\right)^{*}$. Therefore, $\left(\varrho \circ \pi \circ \pi^{-1} \circ \varrho^{-1}\right)^{*} \subset\left(\varrho \circ\left(\pi \circ \pi^{-1}\right)^{*} \circ \varrho^{-1}\right)^{*}$. 
Sufficiency. Suppose that $\left(A, \cdot,{ }^{-1},{ }^{*}, 1, \leq\right)$ satisfies the conditions of Theorem 1. Put $E(A)=\left\{a \in A: a^{*}=a\right\}$.

LEMma 1. $(E(A), \cdot)$ is a semilattice; $1 \in E(A) ;\left(a^{-1}\right)^{*}=a^{*} ;\left(a^{*}\right)^{-1}=a^{*}$; $a^{*} \leq b^{*}$ iff $a^{*} b^{*}=a^{*}$; if $a \leq b^{*}$ then $a \in E(A)$.

It follows from $(3.1)-(3.4)$ that $(E(A), \cdot)$ is a semilattice. Note that $1^{-1}=$ $1 \cdot 1^{-1}=\left(1 \cdot 1^{-1}\right)^{-1}=1$. Since $1=\left(1 \cdot 1^{-1}\right)^{*} \cdot 1=1^{*} \cdot 1=1^{*}$, we have $1 \in E(A)$. Since $\left(a^{*}\right)^{-1}=\left(\left(a^{*}\right)^{-1}\left(\left(a^{*}\right)^{-1}\right)^{-1}\right)^{*}\left(a^{*}\right)^{-1} \leq\left(\left(\left(a^{*}\right)^{-1}\right)^{-1}\right)^{*}=\left(a^{*}\right)^{*}=a^{*}$ and $a^{*}=\left(\left(a^{*}\right)^{-1}\right)^{-1} \leq\left(a^{*}\right)^{-1}$, we have $\left(a^{*}\right)^{-1}=a^{*}$. Since $\left(a^{*}\right)^{-1} \leq a^{-1}$, we have $\left(a^{-1}\right)^{*}=\left(\left(a^{-1}\right)^{*}\right)^{-1} \leq\left(a^{-1}\right)^{-1}=a$, hence $\left(a^{-1}\right)^{*} \leq a^{*}$. Analogously, $a^{*} \leq$ $\left(a^{-1}\right)^{*}$, i.e. $\left(a^{-1}\right)^{*}=a^{*}$.

If $a^{*} b^{*}=a^{*}$ then $a^{*}=a^{*} b^{*} \leq b^{*}$. Conversely, if $a^{*} \leq b^{*}$ then $a^{*}=a^{*} a^{*} \leq$ $a^{*} b^{*}$. Since $a^{*} b^{*} \leq a^{*}$, we have $a^{*} b^{*}=a^{*}$.

Suppose that $a \leq b^{*}$. Then $a^{*} \leq\left(b^{*}\right)^{*}=b^{*}$ and $a=\left(a a^{-1}\right)^{*} a \leq\left(a a^{-1}\right)^{*} b^{*} \leq$ $\left(a\left(b^{*}\right)^{-1}\right)^{*} b^{*} \leq a^{*} b^{*} \leq a^{*}$. Since $a^{*} \leq a$, we have $a^{*}=a$, i.e. $a \in E(A)$. This completes the proof of Lemma 1 .

Define the unary operations $R$ and $L$ as follows: $R a=\left(a a^{-1}\right)^{*} ; L a=\left(a^{-1} a\right)^{*}$. Then $R\left(a^{-1}\right)=L a$ and $R a=a, L a=a$ for each $a \in E(A)$. It follows from (3.5) that $R a a=a$ and $a L a=a$. It follows from (3.6) that $R(a b)=R(a R b)$ and $L(a b)=R\left((a b)^{-1}\right)=R\left(b^{-1} a^{-1}\right)=R\left(b^{-1} R\left(a^{-1}\right)\right)=R\left(b^{-1} L a\right)=R\left((L a b)^{-1}\right)=$ $L(L a b)$.

LEMmA 2. If $L a=R b$ then $R(a b)=R a$ and $L(a b)=L b$.

Indeed, if $L a=R b$ then $R(a b)=R(a R b)=R(a L a)=R a$ and $L(a b)=$ $L(L a b)=L(R b b)=L b$.

For each $B, C \subset A$ we define $B C=\{b c: b \in B \& c \in C\}$ and $B \leq C$ iff $(\forall c \in C)(\exists b \in B) b \leq c$. Note that if $B \subset C$ then $C \leq B$.

Let $\mathbb{N}=\{0,1, \ldots, n, \ldots\}$ and let $f$ be a one-to-one mapping from $\mathbb{N}$ onto $\mathbb{N}^{5}\left(f(k)=\left(n_{k}^{1}, n_{k}^{2}, \ldots, n_{k}^{5}\right)\right)$. Define functions $\varphi, \psi, \alpha, \beta: \mathbb{N} \rightarrow \mathbb{N}$ as follows: $\varphi(k)=n_{k}^{1}$ if $n_{k}^{1} \leq k$, and $\varphi(k)=k$ otherwise; $\psi(k)=n_{k}^{2}$ if $n_{k}^{2} \leq k$, and $\psi(k)=k$ otherwise; $\alpha(k)=n_{k}^{3} ; \beta(k)=n_{k}^{4}$. Clearly, for each $p \in \mathbb{N}$ and $(i, j, m, n) \in \mathbb{N}^{4}$ there exists $k \in \mathbb{N}$ such that $k \geq p$ and $\varphi(k)=i, \psi(k)=j, \alpha(k)=m, \beta(k)=n$.

Suppose that $b_{0}, \ldots, b_{2 n} \in A$. Define subsets $B_{i, j}^{k} \subseteq A$ for $k=1, \ldots, n+1$ and $i, j \leq k$ as follows:

$$
\begin{gathered}
B_{0,1}^{1}=\left\{b_{0}\right\}, \quad B_{1,0}^{1}=\left\{b_{0}^{-1}\right\}, \quad B_{0,0}^{1}=\left\{R b_{0}\right\}, \quad B_{1,1}^{1}=\left\{L b_{0}\right\} \\
B_{i, j}^{k+1}=\bigcup_{p=0}^{k} B_{i, p}^{k} B_{p, j}^{k} ; \\
B_{i, k+1}^{k+1}=B_{i, \varphi(k)}^{k+1}\left\{b_{2 k-1}\right\} \cup B_{i, \psi(k)}^{k+1}\left\{b_{2 k}^{-1}\right\} \\
B_{k+1, i}^{k+1}=\left\{b_{2 k-1}^{-1}\right\} B_{\varphi(k), i}^{k+1} \cup\left\{b_{2 k}\right\} B_{\psi(k), i}^{k+1} ; \\
B_{k+1, k+1}^{k+1}=\left\{L b_{2 k-1} R b_{2 k}\right\} .
\end{gathered}
$$


Lemma 3. $\left(B_{i, j}^{k}\right)^{-1}=B_{j, i}^{k}$ and $B_{i, i}^{i} \subset B_{i, i}^{m}, B_{i, j}^{m} \leq B_{i, j}^{k}$ for $k \leq m$.

According to (D1), $\left(B_{i, j}^{1}\right)^{-1}=B_{j, i}^{1}$. Suppose that $\left(B_{i, j}^{k}\right)^{-1}=B_{j, i}^{k}$. Then

$$
\begin{aligned}
\left(B_{i, j}^{k+1}\right)^{-1} & =\left(\bigcup_{p=0}^{k} B_{i, p}^{k} B_{p, j}^{k}\right)^{-1}=\bigcup_{p=0}^{k}\left(B_{i, p}^{k} B_{p, j}^{k}\right)^{-1} \\
& =\bigcup_{p=0}^{k}\left(B_{p, j}^{k}\right)^{-1}\left(B_{i, p}^{k}\right)^{-1}=\bigcup_{p=0}^{k} B_{j, p}^{k} B_{p, i}^{k}=B_{i, j}^{k+1}, \\
\left(B_{i, k+1}^{k+1}\right)^{-1} & =\left(B_{i, \varphi(k)}^{k+1}\left\{b_{2 k-1}\right\} \cup B_{i, \psi(k)}^{k+1}\left\{b_{2 k}^{-1}\right\}\right)^{-1} \\
& =\left\{b_{2 k-1}^{-1}\right\} B_{\varphi(k), i}^{k+1} \cup\left\{b_{2 k}\right\} B_{\psi(k), i}^{k+1}=B_{k+1, i}^{k+1}, \\
\left(B_{k+1, k+1}^{k+1}\right)^{-1} & =B_{k+1, k+1}^{k+1} .
\end{aligned}
$$

Suppose that $B_{i, i}^{i} \subset B_{i, i}^{m}$. Then $B_{i, i}^{i}=B_{i, i}^{i} B_{i, i}^{i} \subset B_{i, i}^{m} B_{i, i}^{m} \subset B_{i, i}^{m+1}$. If $B_{i, j}^{m} \leq B_{i, j}^{k}$ then $B_{i, j}^{m+1} \leq B_{i, i}^{m} B_{i, j}^{m} \leq B_{i, i}^{i} B_{i, j}^{m} \leq\{1\} B_{i, j}^{m}=B_{i, j}^{m}$.

Since $M\left\{\circ,{ }^{-1},{ }^{*}, \Delta, \subset\right\}$ is a quasivariety [7], without loss of generality we may suppose that $A$ is countable, i.e. $A=\left\{a_{1}, a_{2}, \ldots, a_{n}, \ldots\right\}$.

Using induction for each $d \in A$ we define sequents $b_{0}, \ldots, b_{2 n-1}, b_{2 n}, \ldots$ and $r_{0}, \ldots, r_{n}, \ldots$ of elements of $A$ and $E(A)$ respectively such that $b_{0}=d$ and for all $n$ the following conditions hold:

(a) $B_{\varphi(n), \psi(n)}^{n} \leq\left\{a_{\alpha(n)} a_{\beta(n)}\right\}$;

(b) $R b=r_{i}$ and $L b=r_{j}$ for each $b \in B_{i, j}^{n}$ and $B_{i, i}^{i}=\left\{r_{i}\right\}$.

Base of induction. Put $b_{0}=d$ and $r_{0}=R b_{0}, r_{1}=L b_{0}$.

Inductive step. Suppose that $b_{0}, \ldots, b_{2 m-3}, b_{2 m-2}$ and $r_{0}, \ldots, r_{m}$ have already been defined and (a), (b) are satisfied for $n=1, \ldots, m$ and $i, j \leq m$. Put

$$
\begin{gathered}
b_{2 m-1}=r_{\varphi(m)} a_{\alpha(m)} R\left(a_{\beta(m)} r_{\psi(m)}\right), \\
b_{2 m}=L\left(r_{\varphi(m)} a_{\alpha(m)}\right) a_{\beta(m)} r_{\psi(m)}, \quad r_{m+1}=L b_{2 m-1}
\end{gathered}
$$

if $B_{\varphi(m), \psi(m)}^{m} \leq\left\{a_{\alpha(m)} a_{\beta(m)}\right\}$, and $b_{2 m-1}=b$ for some $b \in B_{\varphi(m), \psi(m)}^{m}, b_{2 m}=$ $r_{\psi(m)}, r_{m+1}=r_{\psi(m)}$, otherwise.

If $b_{2 m-1}=b$ for some $b \in B_{\varphi(m), \psi(m)}^{m}, b_{2 m}=r_{\psi(m)}, r_{m+1}=r_{\psi(m)}$ then $b=b L b=b r_{\psi(m)}=b_{2 m-1} b_{2 m}$, i.e. $B_{\varphi(m), \psi(m)}^{m} \leq b_{2 m-1} b_{2 m}$ and $L b_{2 m-1}=$ $R b_{2 m}=r_{m+1}$, hence $B_{m+1, m+1}^{m+1}=\left\{L b_{2 m-1} R b_{2 m}\right\}=\left\{r_{m+1}\right\}$. then

If $B_{\varphi(m), \psi(m)}^{m} \leq\left\{a_{\alpha(m)} a_{\beta(m)}\right\}$, i.e. $b \leq a_{\alpha(m)} a_{\beta(m)}$ for some $b \in B_{\varphi(m), \psi(m)}^{m}$,

$$
\begin{aligned}
b & =r_{\varphi(m)} b r_{\psi(m)} \leq r_{\varphi(m)} a_{\alpha(m)} a_{\beta(m)} r_{\psi(m)} \\
& =r_{\varphi(m)} a_{\alpha(m)} L\left(r_{\varphi(m)} a_{\alpha(m)}\right) R\left(a_{\beta(m)} r_{\psi(m)}\right) a_{\beta(m)} r_{\psi(m)} \\
& =r_{\varphi(m)} a_{\alpha(m)} R\left(a_{\beta(m)} r_{\psi(m)}\right) L\left(r_{\varphi(m)} a_{\alpha(m)}\right) a_{\beta(m)} r_{\psi(m)}=b_{2 m-1} b_{2 m},
\end{aligned}
$$

i.e. $B_{\varphi(m), \psi(m)}^{m} \leq b_{2 m-1} b_{2 m}$. 
Since $b \leq b_{2 m-1} b_{2 m}$ for some $b \in B_{\varphi(m), \psi(m)}^{m}$, we have

$$
r_{\varphi(m)}=R b \leq R\left(b_{2 m-1} b_{2 m}\right) \leq R\left(b_{2 m-1} R b_{2 m}\right) \leq R\left(b_{2 m-1}\right) .
$$

On the other hand,

$$
\begin{aligned}
R b_{2 m-1} & =R\left(r_{\varphi(m)} a_{\alpha(m)} R\left(a_{\beta(m)} r_{\psi(m)}\right)\right) \\
& =R\left(r_{\varphi(m)} R\left(a_{\alpha(m)} R\left(a_{\beta(m)} r_{\psi(m)}\right)\right)\right) \leq R\left(r_{\varphi(m)}\right)=r_{\varphi(m)},
\end{aligned}
$$

hence $R b_{2 m-1}=r_{\varphi(m)}$. Analogously, $R b_{2 m}=r_{\psi(m)}$. Since $r_{m+1}=L b_{2 m-1}$, we have

$$
\begin{aligned}
r_{m+1} & =L b_{2 m-1}=L\left(r_{\varphi(m)} a_{\alpha(m)} R\left(a_{\beta(m)} r_{\psi(m)}\right)\right) \\
& =L\left(L\left(r_{\varphi(m)} a_{\alpha(m)}\right) R\left(a_{\beta(m)} r_{\psi(m)}\right)\right)=R\left(L\left(r_{\varphi(m)} a_{\alpha(m)}\right) R\left(a_{\beta(m)} r_{\psi(m)}\right)\right) \\
& =R\left(L\left(r_{\varphi(m)} a_{\alpha(m)}\right) a_{\beta(m)} r_{\psi(m)}\right)=R b_{2 m} .
\end{aligned}
$$

Therefore, using Lemma 2 and the definition (D1)-(D4) we conclude that (b) is satisfied for $i, j \leq m+1$.

Put $B_{i, j}=\bigcup\left\{B_{i, j}^{n}: n \in \mathbb{N}\right\}$. Then $B_{i, k} B_{k, j} \leq B_{i, j}$.

LEMMA 4. $\left\{b_{0}\right\} \leq B_{0,1}$ and $\left\{r_{i}\right\} \leq B_{i, i}$.

Note that if $b \in B_{i, j}$ then $b$ can be represented as a product of elements $b_{1}, \ldots, b_{m}, \ldots$ and $b_{0}^{-1}, \ldots, b_{m}^{-1}, \ldots$ This product constructed according to (D1)(D4) will be called the canonical form of $b$. Let $Q_{k}(b)$ be the number of occurrences of elements $a_{2 k-1}, a_{2 k}$ in the canonical form of $b$ and $Q(b)=\max \left\{k: Q_{k}(b)>0\right\}$.

Suppose that $b \in B_{0,1}$. If $Q(b)=0$ then according to (D1)-(D4), $b=$ $b_{0}\left(b_{0}^{-1} b_{0}\right)^{m}$ for some $m$ and we have $b_{0}=b_{0} L b_{0}=b_{0}\left(b_{0}^{-1} b_{0}\right)^{*} \leq b_{0} b_{0}^{-1} b_{0} \leq$ $\ldots \leq b_{0}\left(b_{0}^{-1} b_{0}\right)^{m}=b$. Assume that for every $b \in B_{0,1}$ if $Q(b) \leq k$ and $Q_{k}(b)=p$ then $b_{0} \leq b$. Suppose that $Q_{k}(b)=p+1$. According to (D1)-(D4) the following cases are possible:

1) $b=c_{1} b_{2 k-1} b_{2 k} c_{2}$ where $c_{1} \in B_{0, \varphi(k)}$ and $c_{2} \in B_{\psi(k), 1}$. Since $B_{\varphi(k), \psi(k)}^{k} \leq$ $\left\{b_{2 k-1} b_{2 k}\right\}$, i.e. $c \leq\left\{b_{2 k-1} b_{2 k}\right\}$ for some $c \in B_{\varphi(k), \psi(k)}^{k}$, using the inductive assumption we have $b_{0} \leq c_{1} c c_{2} \leq c_{1} b_{2 k-1} b_{2 k} c_{2}=b$.

2) $b=c_{1} b_{2 k}^{-1} b_{2 k-1}^{-1} c_{2}$ where $c_{1} \in B_{0, \psi(k)}$ and $c_{2} \in B_{\varphi(k), 1}$. This case is analogous to Case 1 .

3) $b=c_{1} b_{2 k-1} b_{2 k-1}^{-1} c_{2}$ where $c_{1} \in B_{0, \varphi(k)}$ and $c_{2} \in B_{\varphi(k), 1}$. Since $L c_{1}=$ $R b_{2 k-1}=r_{\varphi(k)}$, using the inductive assumption we have $b_{0} \leq c_{1} c_{2}=c_{1} L c_{1} c_{2}=$ $c_{1} R b_{2 k-1} c_{2}=c_{1}\left(b_{2 k-1} b_{2 k-1}^{-1}\right)^{*} c_{2} \leq c_{1} b_{2 k-1} b_{2 k-1}^{-1} c_{2}=b$.

4) $b=c_{1} b_{2 k}^{-1} b_{2 k} c_{2}$ where $c_{1} \in B_{0, \psi(k)}$ and $c_{2} \in B_{\psi(k), 1}$. This case is analogous to Case 3.

Suppose that $b \in B_{i, i}$. If $Q(b)=0$ then according to (D1)-(D4), $i=0$ or $i=1$. If $i=0$ then $b=r_{0}$ or $b=\left(b_{0} b_{0}^{-1}\right)^{m}$ for some $m$ and we have $r_{0}=\left(r_{0}\right)^{m}=$ $\left(R b_{0}\right)^{m}=\left(\left(b_{0} b_{0}^{-1}\right)^{*}\right)^{m} \leq\left(b_{0} b_{0}^{-1}\right)^{m}$. The case $i=1$ is analogous. Assume that for every $b \in B_{i, i}$ if $Q(b) \leq k$ and $Q_{k}(b)=p$ then $r_{i} \leq b$. Suppose that $Q_{k}(b)=p+1$. According to (D1)-(D4) the following cases are possible: 
1) $b=c_{1} b_{2 k-1} b_{2 k} c_{2}$ where $c_{1} \in B_{i, \varphi(k)}$ and $c_{2} \in B_{\psi(k), i}$. Since $B_{\varphi(k), \psi(k)}^{k} \leq$ $\left\{b_{2 k-1} b_{2 k}\right\}$, i.e. $c \leq\left\{b_{2 k-1} b_{2 k}\right\}$ for some $c \in B_{\varphi(k), \psi(k)}^{k}$, using the inductive assumption we have $r_{i} \leq c_{1} c c_{2} \leq c_{1} b_{2 k-1} b_{2 k} c_{2}=b$.

2) $b=c_{1} b_{2 k}^{-1} b_{2 k-1}^{-1} c_{2}$ where $c_{1} \in B_{i, \psi(k)}$ and $c_{2} \in B_{\varphi(k), i}$. This case is analogous to Case 1.

3) $b=c_{1} b_{2 k-1} b_{2 k-1}^{-1} c_{2}$ where $c_{1} \in B_{i, \varphi(k)}$ and $c_{2} \in B_{\varphi(k), i}$. Since $L c_{1}=$ $R b_{2 k-1}=r_{\varphi(k)}$, using the inductive assumption we have $r_{i} \leq c_{1} c_{2}=c_{1} L c_{1} c_{2}=$ $c_{1} R b_{2 k-1} c_{2}=c_{1}\left(b_{2 k-1} b_{2 k-1}^{-1}\right)^{*} c_{2} \leq c_{1} b_{2 k-1} b_{2 k-1}^{-1} c_{2}=b$.

4) $b=c_{1} b_{2 k}^{-1} b_{2 k} c_{2}$ where $c_{1} \in B_{i, \psi(k)}$ and $c_{2} \in B_{\psi(k), i}$. This case is analogous to Case 3.

This completes the proof of Lemma 3.

Define the mapping $F_{d}: A \rightarrow \operatorname{Rel}(\mathbb{N})$ as follows:

$$
F_{d}(a)=\left\{(i, j): B_{i, j} \leq\{a\}\right\} .
$$

Since $\left(B_{i, j}\right)^{-1}=B_{j, i}$, we have $F_{d}\left(a^{-1}\right)=\left(F_{d}(a)\right)^{-1}$. Obviously, $a \leq b$ implies $F_{d}(a) \subset F_{d}(b)$.

We show that $F_{d}(a b)=F_{d}(a) \circ F_{d}(b)$. If $(i, j) \in F_{d}(a) \circ F_{d}(b)$, i.e. $(i, k) \in F_{d}(a)$ and $(k, j) \in F_{d}(b)$ for some $k$, then $B_{i, k} \leq\{a\}$ and $B_{k, j} \leq\{b\}$, hence $B_{i, j} \leq$ $B_{i, k} B_{k, j} \leq\{a b\}$, i.e. $(i, j) \in F_{d}(a b)$. Conversely, suppose that $(i, j) \in F_{d}(a b)$, i.e. $B_{i, j} \leq\{a b\}$. Then $B_{i, j}^{p} \leq\{a b\}$ for some $p$ and there exists $k \geq p$ such that $\varphi(k)=i, \psi(k)=j, a=a_{\varphi(k)}, b=a_{\psi(k)}$. Since $B_{\varphi(k), \psi(k)}^{k} \leq B_{\varphi(k), \psi(k)}^{p}=$ $B_{i, j}^{p} \leq\{a b\}=\left\{a_{\alpha(k)} a_{\beta(k)}\right\}$, we have $b_{2 m-1}=r_{\varphi(m)} a_{\alpha(m)} R\left(a_{\beta(m)} r_{\psi(m)}\right), b_{2 m}=$ $L\left(r_{\varphi(m)} a_{\alpha(m)}\right) b_{\beta(m)} r_{\psi(m)}, r_{m+1}=L b_{2 m-1}$, hence $b_{2 k-1} \leq a$ and $b_{2 k} \leq b$. Since $b_{2 k-1}=r_{i} b_{2 k-1} \in B_{i, \varphi(k)}^{k} b_{2 k-1} \subset B_{i, k+1}^{k+1}$, we have $(i, k+1) \in F_{d}\left(b_{2 k-1}\right) \subset F_{d}(a)$. Analogously, $(k+1, j) \in F_{d}\left(b_{2 k}\right) \subset F_{d}(b)$. Thus, $(i, j) \in F_{d}(a) \circ F_{d}(b)$.

We show that $F_{d}\left(a^{*}\right)=F_{d}(a) \cap F_{d}(1)$. Since $a^{*} \leq a$ and $a^{*} \leq 1$, we have $F_{d}\left(a^{*}\right) \subset F_{d}(a)$ and $F_{d}\left(a^{*}\right) \subset F_{d}(1)$. Conversely, suppose that $(i, j) \in F_{d}(a) \cap$ $F_{d}(1)$; then $B_{i, j} \leq\{a\}$ and $B_{i, j} \leq\{1\}$, hence $B_{i, i} \leq B_{i, j}\left(B_{i, j}\right)^{-1} \leq\left\{a 1^{-1}\right\}=\{a\}$. Since $\left\{r_{i}\right\} \leq B_{i, i}$, we have $r_{i} \leq a$, hence $r_{i}=r_{i}^{*} \leq a^{*}$. Since $r_{i} \in B_{i, i}$, we have $(i, i) \in F_{d}\left(r_{i}\right) \subset F_{d}\left(a^{*}\right)$. It now follows from $(i, i) \in F_{d}\left(a^{*}\right)$ and $(i, j) \in F_{d}(1)$ that $(i, j) \in F_{d}\left(a^{*}\right) \circ F_{d}(1)=F_{d}\left(a^{*} 1\right)=F_{d}\left(a^{*}\right)$.

Put $X_{d}=X \times\{d\}$ and $X=\bigcup\left\{X_{d}: d \in A\right\}, F_{d}^{0}(a)=\{((i, d),(j, d))$ : $\left.(i, j) \in F_{d}(a)\right\}$ and $F(a)=\bigcup\left\{F_{d}^{0}(a): d \in A\right\}$. Obviously $F(a b)=F(a) \circ F(b)$, $F(a)^{-1}=F(a)^{-1}, F\left(a^{*}\right)=F(a) \cap F(1)$, and $a \leq b$ implies $F(a) \subset F(b)$. Suppose that $F(a) \subset F(b)$; then $F_{a}(a) \subset F_{a}(b)$. Since $(0,1) \in F_{a}(a)$, we have $(0,1) \in$ $F_{a}(b)$, i.e. $B_{0,1} \leq\{b\}$. Then using Lemma 4 , we obtain $\{a\} \leq B_{0,1} \leq\{b\}$, i.e. $a \leq b$. Therefore, $F$ is an isomorphism of $\left(A, \cdot,^{-1}, \leq\right)$ into $\left(\operatorname{Rel}(X), \circ,{ }^{-1}, \subset\right)$ and $F\left(a^{*}\right)=F(a) \cap F(1)$.

It is clear that $\varepsilon=F(1)$ is an equivalence relation on $X$. Let $Y=X / \varepsilon$ and let $\eta$ be the natural mapping of $X$ onto $Y$. Put $P(a)=\eta \circ F(a) \circ \eta^{-1}$. It is easy to see that $P$ is an isomorphism of $\left(A, \cdot,^{-1}, \leq\right)$ into $\left(\operatorname{Rel}(Y),{ }^{-1}, \subset\right)$ and $P(1)=\Delta$. It 
follows that $P\left(a^{*}\right)=P(a) \cap P(1)=P(a) \cap \Delta=P(a)^{*}$. This completes the proof of Theorem 1.

Proof of Theorems 2 and 3 . Suppose that $\left(A, \cdot,{ }^{-1}, \wedge, 1\right)$ is a semilattice ordered involuted monoid and (4) holds. Let $\leq$ be the canonical order relation of the semilattice $(A, \wedge)$. Put $a^{*}=a \wedge 1$. Obviously, $a^{*} \leq 1$ and $a^{*} \leq a$.

Lemma 5. The operations $\cdot{ }^{-1},{ }^{*}$ are monotonic.

If $a \leq b$, i.e. $a \wedge b=a$, then $a^{-1} \wedge b^{-1}=(a \wedge b)^{-1}=a^{-1}$, i.e. $a^{-1} \leq b^{-1}$, and $a^{*}=a \wedge 1 \leq b \wedge 1=b^{*}$. Also if $a \leq b$, i.e. $a \wedge b=a$, then $a c=(a \wedge b) c \leq a c \wedge b c \leq b c$ and $c(a \wedge b) \leq c a \wedge c b \leq c b$.

Lemma 6. $x y \wedge z \leq x\left(y \wedge x^{-1} z\right), x \leq x x^{-1} x$.

Indeed, $x y \wedge z=\left(y^{-1} x^{-1} \wedge z^{-1}\right)^{-1} \leq\left(\left(y^{-1} \wedge z^{-1} x\right) x^{-1}\right)^{-1}=x(y \wedge x z)$, $x=x 1 \wedge x \leq x\left(1 \wedge x^{-1} x\right) \leq x x^{-1} x$.

LEMma 7. $\left(x^{-1}\right)^{*}=x^{*}, x^{*} x^{*}=x^{*},\left(x^{*}\right)^{-1}=x^{*}$.

Indeed, $x^{*}=x \wedge 1=(x \wedge 1) 1 \wedge 1 \leq(x \wedge 1)\left(1 \wedge(x \wedge 1)^{-1} 1\right) \leq(x \wedge 1)^{-1}=$ $x^{-1} \wedge 1=\left(x^{-1}\right)^{*}$ and $\left(x^{-1}\right)^{*} \leq\left(\left(x^{-1}\right)^{-1}\right)^{*}=x^{*}$. The second assertion follows from

$$
x^{*} x^{*}=(x \wedge 1)(x \wedge 1) \leq(x \wedge 1) 1 \leq x \wedge 1=x^{*}
$$

and

$$
\begin{aligned}
x^{*} & =x \wedge 1=(x \wedge 1) 1 \wedge 1 \leq(x \wedge 1)\left(1 \wedge(x \wedge 1)^{-1} 1\right) \\
& \leq(x \wedge 1)\left(1 \wedge x^{-1}\right)=x^{*}\left(x^{-1}\right)^{*}=x^{*} x^{*} .
\end{aligned}
$$

Finally, $\left(x^{*}\right)^{-1}=(x \wedge 1)^{-1}=x^{-1} \wedge 1^{-1}=x^{-1} \wedge 1=\left(x^{-1}\right)^{*}=x^{*}$.

Lemma 8. $x^{*} y^{*}=x^{*} \wedge y^{*}, x^{*} y^{*}=y^{*} x^{*},\left(x^{*} y^{*}\right)^{*}=x^{*} y^{*}$

Since $x^{*} y^{*} \leq x^{*} 1=x^{*}$ and $x^{*} y^{*} \leq 1 y^{*}=y^{*}$, we have $x^{*} y^{*} \leq x^{*} \wedge y^{*}$. Conversely, $x^{*} \wedge y^{*}=x^{*} 1 \wedge y^{*} \leq x^{*}\left(1 \wedge\left(x^{*}\right)^{-1} y^{*}\right) \leq x^{*} x^{*} y^{*}=x^{*} y^{*}$. Thus, $x^{*} y^{*}=x^{*} \wedge y^{*}$. It follows that $x^{*} y^{*}=x^{*} \wedge y^{*}=y^{*} \wedge x^{*}=y^{*} x^{*}$ and $\left(x^{*} y^{*}\right)^{*}=$ $x^{*} y^{*} \wedge 1=x^{*} \wedge y^{*} \wedge 1=x^{*} \wedge y^{*}=x^{*} y^{*}$.

Lemma 9. $\left(x y y^{-1} x^{-1}\right)^{*} \leq\left(x x^{-1}\right)^{*},\left(x x^{-1}\right)^{*} x=x$.

Indeed, $\left(x y y^{-1} x^{-1}\right)^{*}=x y y^{-1} x^{-1} \wedge 1=\left(x y y^{-1} x^{-1} \wedge 1\right) \wedge 1 \leq x\left(y y^{-1} x^{-1} \wedge\right.$ $\left.x^{-1} 1\right) \wedge 1 \leq x x^{-1} \wedge 1=\left(x x^{-1}\right)^{*}$. For the second assertion, $\left(x x^{-1}\right)^{*} x \leq 1 x=x$ and $x=1 x \wedge x \leq\left(1 \wedge x x^{-1}\right) x=\left(x x^{-1}\right)^{*} x$.

LEMma 10. $\left(x y y^{-1} x^{-1}\right)^{*}=\left(x\left(y y^{-1}\right)^{*} x^{-1}\right)^{*}$.

Indeed,

$$
\begin{aligned}
\left(x y y^{-1} x^{-1}\right)^{*} & =\left(x\left(\left(y y^{-1}\right)^{*} y\right)\left(\left(y y^{-1}\right)^{*} y\right)^{-1} x^{-1}\right)^{*} \\
& =\left(x\left(y y^{-1}\right)^{*} y y^{-1}\left(\left(y y^{-1}\right)^{*}\right)^{-1} x^{-1}\right)^{*} \\
& \leq\left(x\left(y y^{-1}\right)^{*}\left(\left(y y^{-1}\right)^{*}\right)^{-1} x^{-1}\right)^{*} \\
& =\left(x\left(y y^{-1}\right)^{*}\left(y y^{-1}\right)^{*} x^{-1}\right)^{*}=\left(x\left(y y^{-1}\right)^{*} x^{-1}\right)^{*}
\end{aligned}
$$


and

$$
\left(x\left(y y^{-1}\right)^{*} x^{-1}\right)^{*} \leq\left(x y y^{-1} x^{-1}\right)^{*} .
$$

According to Lemmas $5-10,\left(A, \cdot,^{-1},{ }^{*}, 1, \leq\right)$ satisfies the conditions of Theorem 1 . This immediately implies the conclusion of Theorem 2 .

Lemma 11. $x \wedge y(z \wedge t z) \leq y\left(y^{-1} x z^{-1} \wedge t\right) z$.

Indeed,

$$
\begin{aligned}
& y(z \wedge t z) \wedge x \leq y\left(z \wedge t z \wedge y^{-1} x\right) \\
& \quad \leq y\left(\left(t \wedge z z^{-1}\right) z \wedge y^{-1} x\right) \leq y\left(t \wedge z z^{-1} \wedge y^{-1} x z^{-1}\right) z \leq y\left(t \wedge y^{-1} x z^{-1}\right) z .
\end{aligned}
$$

Lemma 12. If $x^{-1} x \leq 1$ then $x(y \wedge z)=x y \wedge x z$ and $(y \wedge z) x^{-1}=y x^{-1} \wedge z x^{-1}$.

Indeed, $x(y \wedge z) \leq x y \wedge x z$ and $x y \wedge x z \leq x\left(y \wedge x^{-1} x z\right) \leq x(y \wedge 1 z)=x(y \wedge z)$. The proof of the second assertion is similar.

Lemma 13. If $u^{-1} u \leq 1, v^{-1} v \leq 1$ and $x \wedge y \leq u^{-1} v$, then $x \wedge y=$ $u^{-1}\left(u x v^{-1}\right)^{*}\left(u y v^{-1}\right)^{*} v$.

Since $u^{-1} u \leq 1$ and $v^{-1} v \leq 1$, we have $u^{-1}\left(u x v^{-1}\right)^{*}\left(u y v^{-1}\right)^{*} v \leq$ $u^{-1}\left(u x v^{-1}\right)^{*} v \leq u^{-1} u x v^{-1} v \leq x$. Analogously, $u^{-1}\left(u x v^{-1}\right)^{*}\left(u y v^{-1}\right)^{*} v \leq y$. Thus, $u^{-1}\left(u x v^{-1}\right)^{*}\left(u y v^{-1}\right)^{*} v \leq x \wedge y$. Conversely, using Lemmas 11, 12, we obtain

$$
\begin{aligned}
x \wedge y & =x \wedge y \wedge u^{-1} v \\
& =x \wedge y \wedge u^{-1}(v \wedge 1 v)=u^{-1}\left(u(x \wedge y) v^{-1} \wedge 1\right) v \\
& =u^{-1}\left(u x v^{-1} \wedge u y v^{-1} \wedge 1\right) v=u^{-1}\left(\left(u x v^{-1} \wedge 1\right) \wedge\left(u y v^{-1} \wedge 1\right)\right) v \\
& =u^{-1}\left(\left(u x v^{-1}\right)^{*} \wedge\left(u y v^{-1}\right)^{*}\right) v=u^{-1}\left(\left(u x v^{-1}\right)^{*}\left(u y v^{-1}\right)^{*}\right) v .
\end{aligned}
$$

Lemma 14. Suppose that $\varrho, \pi, \alpha, \beta \in \operatorname{Rel}(X)$ and $\alpha^{-1} \circ \alpha \subset \Delta, \beta^{-1} \beta \subset \Delta$, $\varrho \cap \pi \subset \alpha^{-1} \circ \beta$. Then

$$
\varrho \cap \pi=\alpha^{-1} \circ\left(\alpha \circ \varrho \circ \beta^{-1}\right)^{*} \circ\left(\alpha \circ \pi \circ \beta^{-1}\right)^{*} \circ \beta .
$$

It follows from $\alpha^{-1} \circ \alpha \subset \Delta$ and $\beta^{-1} \circ \beta \subset \Delta$ that $\alpha, \beta$ are functions. Therefore, we write $y=\alpha(x)$ and $y=\beta(x)$ instead of $(x, y) \in \alpha$ and $(x, y) \in \beta$. Since $\varrho \cap \pi \subset \alpha^{-1} \circ \beta$, for each pair $(x, y) \in \varrho \cap \pi$ there exists $z \in X$ such that $\alpha(z)=x$ and $\beta(z)=y$.

Suppose that $(x, y) \in \alpha^{-1} \circ\left(\alpha \circ \varrho \circ \beta^{-1}\right)^{*} \circ\left(\alpha \circ \pi \circ \beta^{-1}\right)^{*} \circ \beta$. Then $x=\alpha(z)$ and $y=\beta(z)$ for some $z$ such that $(z, z) \in\left(\alpha \circ \varrho \circ \beta^{-1}\right)^{*} \circ\left(\alpha \circ \pi \circ \beta^{-1}\right)^{*}$. It follows that $(z, z) \in \alpha \circ \varrho \circ \beta^{-1}$ and $(z, z) \in \alpha \circ \pi \circ \beta^{-1}$, hence $(x, y)=(\alpha(z), \beta(z)) \in \varrho \cap \pi$. Conversely, let $(x, y) \in \varrho \cap \pi$. Since $x=\alpha(z)$ and $y=\beta(z)$ for some $z$, we have $(z, z) \in\left(\alpha \circ \varrho \circ \beta^{-1}\right)^{*}$ and $(z, z) \in\left(\alpha \circ \pi \circ \beta^{-1}\right)^{*}$, hence $(z, z) \in\left(\alpha \circ \varrho \circ \beta^{-1}\right)^{*} \circ$ $\left(\alpha \circ \pi \circ \beta^{-1}\right)^{*}$ and $(x, y)=(\alpha(z), \beta(z)) \in \alpha^{-1} \circ\left(\alpha \circ \varrho \circ \beta^{-1}\right)^{*} \circ\left(\alpha \circ \pi \circ \beta^{-1}\right)^{*} \circ \beta$, which completes the proof of Lemma 14 .

Theorem 2 and Lemmas 13, 14 immediately imply Theorem 3. 


\section{References}

[1] D. A. Bredikhin, Representation of ordered involuted semigroups, Izv. Vyssh. Uchebn. Zaved. Mat. 7 (1975), 19-29 (in Russian).

[2] - , Abstract characteristics of some relation algebras, in: Algebra and Number Theory, Nalchic 1977, 3-19 (in Russian).

[3] L. H. Chin and A. Tarski, Distributive and modular laws in the arithmetic of relation algebras, Univ. Calif. Publ. Math. 1 (1951), 341-383.

[4] S. A. Givant and A. Tarski, A formalization of set theory without variables, Amer. Math. Soc. Colloq. Publ. 41, Amer. Math. Soc., Providence, R.I., 1987.

[5] M. Haivan, Arguesian lattices which are not linear, Bull. Amer. Math. Soc. 16 (1987), 121-123.

[6] B. Jónsson, Representation of modular lattices and of relation algebras, Trans. Amer. Math. Soc. 92 (1959), 449-464.

[7] B. M. Schein, Relation algebras and function semigroups, Semigroup Forum 1 (1970), $1-62$.

[8] - Representation of involuted semigroups by binary relations, Fund. Math. 82 (1974), $121-141$. 